\title{
Natural convection in the cavity with the differentially heated isoflux boundaries
}

\section{S. Jiracheewanun ${ }^{1}$ \\ G. D. McBain ${ }^{2}$ \\ S. W. Armfield ${ }^{3}$ \\ M. Behnia ${ }^{4}$}

(Received 30 August 2006; revised 15 February 2008)

\begin{abstract}
Natural convection flow in rectangular cavities with uniform heat flux side walls and an adiabatic floor and ceiling is investigated. The analytical solution for the evenly heated and cooled infinitely tall cavity, obtained by integrating the energy equation over a certain control volume, is introduced and compared to a full numerical solution for the finite cavity. Numerical solutions have been obtained for cavities with height-to-width ratios of 1 to 10, various values of the heat flux and with parameters appropriate to both air and water. For high enough aspect ratio and/or Rayleigh number the numerical solutions at mid-height of the cavity and the cavity stratification are well predicted by the analytical solution for the infinite cavity. The flow on the cavity side walls is then one dimensional.
\end{abstract}

See http://anziamj.austms.org.au/ojs/index.php/ANZIAMJ/article/view/113 for this article, (c) Austral. Mathematical Soc. 2008. Published March 23, 2008. ISSN 1446-8735 


\section{Contents}

1 Introduction

C978

2 Mathematical model

C979

3 Analytical solution for evenly heated slot

C981

4 Temperature and velocity profiles

C982

5 Conclusions

C987

References

C987

\section{Introduction}

Natural convection in a rectangular cavity has been extensively studied because of its wide range of engineering applications, such as double glazed windows and electronic cooling systems. In previous studies, the cavity with fixed uniform temperature side walls has been examined for various configurations (square, shallow and tall cavity) using experimental and numerical approaches $[1,2,10,11]$. On the other hand, cavities with isoflux side walls have received far less attention, although the uniform heat flux configurations are more appropriate for many real systems, which often include radiative heating and cooling, than the uniform temperature model. Kimura and Bejan [5] are among the first who studied the cavity with the isoflux side walls. The flow features for the isoflux cavity have been investigated $[4,5]$ and it was found that the transient flow features are similar to the flow features obtained for the isothermal cavity. However, at full development, the isoflux cavity has a uniform boundary layer thickness on the side walls and flow only in the vicinity of the boundaries, with a stratified quiescent core $[3,4,5]$. Kimura and Bejan [5] also derived analytical solutions for the velocity and 


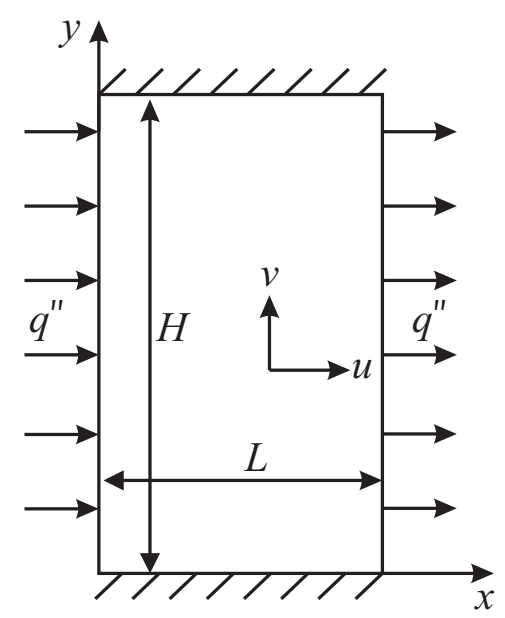

Figure 1: Computational domain and coordinate system.

temperature in the boundary layers. Desrayaud and Nguyen [3] applied the same energy balance as Kimura and Bejan to Lietzke's [7] analytical fully developed solutions for an infinite slot and determined the stratification appropriate to them. These solutions are valid for high aspect ratio cavities; however, their validity for lower aspect ratios is not known. The objective of this study is to determine the range of Rayleigh numbers and aspect ratios for which these analytical solutions provide a satisfactory prediction of the full solution. Numerical results of temperature and velocity for the isoflux cavity are compared directly to the analytical solution for different Rayleigh numbers, Prandtl numbers and aspect ratios.

\section{Mathematical model}

The governing equations are the two dimensional Navier-Stokes and energy equations using the Boussinesq approximation for buoyancy. The equations are written in non-dimensional form as 


$$
\begin{aligned}
\frac{\partial u}{\partial x}+\frac{\partial v}{\partial y} & =0 \\
\frac{\partial u}{\partial t}+u \frac{\partial u}{\partial x}+v \frac{\partial u}{\partial y} & =-\frac{\partial p}{\partial x}+\frac{\partial^{2} u}{\partial x^{2}}+\frac{\partial^{2} u}{\partial y^{2}}, \\
\frac{\partial v}{\partial t}+u \frac{\partial v}{\partial x}+v \frac{\partial v}{\partial y} & =-\frac{\partial p}{\partial y}+\frac{\partial^{2} v}{\partial x^{2}}+\frac{\partial^{2} v}{\partial y^{2}}+\frac{\operatorname{Ra}}{\operatorname{Pr}} T, \\
\frac{\partial T}{\partial t}+u \frac{\partial T}{\partial x}+v \frac{\partial T}{\partial y} & =\frac{1}{\operatorname{Pr}}\left(\frac{\partial^{2} T}{\partial x^{2}}+\frac{\partial^{2} T}{\partial y^{2}}\right),
\end{aligned}
$$

where $u$ and $v$ are the velocity components in the $x$ - and $y$-direction as shown in Figure 1, $t$ is time, $p$ is pressure and $T$ is temperature. In these equations, velocity is non-dimensionalised by $U=\nu / L$, length by $L$, time by $L^{2} / \nu$, pressure by $\rho(\nu / L)^{2}$, and $T-T_{0}$ by $q^{\prime \prime} L / k$. Prandtl number (ratio of momentum diffusivity and thermal diffusivity) and Rayleigh number (ratio of the buoyancy force to the viscous force) are defined as

$$
\begin{aligned}
\operatorname{Pr} & =\frac{\nu}{\alpha}, \\
\operatorname{Ra} & =\frac{g \beta q^{\prime \prime} L^{4}}{\alpha \nu k},
\end{aligned}
$$

where $\beta$ is the coefficient of volume expansion, $q^{\prime \prime}$ is heat flux, $\alpha$ is the thermal diffusivity, $\nu$ is the kinematic viscosity, $k$ is the thermal conductivity and $L$ is the cavity width. The computational domain is $0<x<1,0<y<A$, shown in Figure 1, and the corresponding dimensionless initial and boundary conditions are

$$
\begin{aligned}
& T=u=v=0 \text { at all } x, y \text { and } t<0, \\
& \frac{\partial T}{\partial x}=-1 \text { on } x=0,1, \\
& \frac{\partial T}{\partial y}=0 \text { on } y=0, A, \\
& u=v=0 \text { on } x=0,1 \text { and } y=0, A,
\end{aligned}
$$


where $A$ is the height-to-width ratio $(H / L)$. The numerical simulations have been carried out using a finite volume method. The governing equations are discretized on a non-staggered mesh, with standard second order, central differencing used for the viscous, pressure gradient, and divergence terms, whereas the QUICK, third order, upwind scheme [6] is used for the advective terms. The momentum and temperature equations are solved using the biconjugate gradient stabilized method. The second order Adams-Bashforth and Crank-Nicolson schemes are used for the time integration of the advective and the diffusive terms, respectively. To enforce continuity, the noniterative, fractional step, pressure correction method is used to construct a Poisson equation, which is solved using the bi-conjugate gradient stabilized method $[1,2,9,10]$. A non-uniform mesh is used with the smallest grid size, near the boundaries, of 0.005 . The grid size increases away from the boundaries, with a stretching rate of $7 \%$, producing a coarser grid in the cavity interior. For testing the isoflux cavity with $\mathrm{Ra}=10^{3}$, 66 nodes are used in the $x$-direction and 66, 84, 110 and 130 nodes in the $y$-direction for the aspect ratios of $1,2,5$ and 10 , respectively.

\section{Analytical solution for evenly heated slot}

Lietzke [7] obtained solutions for the velocity and temperature fields in the evenly heated slot with specified stratification, that is equations (1)-(4) with heating and cooling on the vertical walls as given above but in an infinite vertical domain and with the temperature gradient constant in the $y$-direction. The non-dimensional form of these solutions with the stratified background temperature are

$$
\begin{aligned}
v(x) & =\frac{\operatorname{Ra}}{2 \operatorname{Pr}} \frac{\sinh [\gamma(1-x)] \sin (\gamma x)-\sinh (\gamma x) \sin [\gamma(1-x)]}{\gamma^{3}(\sinh \gamma+\sin \gamma)}, \\
T(x) & =\frac{\cosh [\gamma(1-x)] \cos (\gamma x)-\cosh (\gamma x) \cos [\gamma(1-x)]}{\gamma(\sinh \gamma+\sin \gamma)}+\frac{4 \gamma^{4} y}{\mathrm{Ra}},
\end{aligned}
$$


where $\gamma$ is the stratification parameter which is related to the non-dimensional stratification

$$
\Gamma_{s}=\frac{\partial T}{\partial y}=\frac{4 \gamma^{4}}{\mathrm{Ra}} .
$$

By combining Lietzke's one dimensional solution assumption with Kimura and Bejan's energy control volume, the relation between $\mathrm{Ra}$ and $\gamma$ in the cavity is $[3,8]$

$$
\operatorname{Ra}^{2}=\frac{32 \gamma^{9}(\sinh \gamma+\sin \gamma)^{2}}{(\sinh \gamma+\sin \gamma)(\cosh \gamma-\cos \gamma)-2 \gamma \sinh \gamma \sin \gamma}
$$

Thus, we combine (7), (8) and (10) to obtain the temperature and vertical velocity distribution along the $x$ axis, and with (9) to obtain the stratification for the cavity flow.

\section{Temperature and velocity profiles}

The solutions given in equations (7) and (8) are compared to the numerical results with various aspect ratios from $A=1$ to 10 with a fixed low Rayleigh number $\mathrm{Ra}=10^{3}$ for Pr of 0.7 (air) and 7.5 (water). In Figure 2 the comparison of temperature and velocity profiles from the hot wall at the mid-height of the cavity shows that the analytical solutions for the evenly heated slot are not valid for the low aspect ratio cavities $(A=1-2)$. For aspect ratios of $5-10$, the numerical solutions agree with the analytical solutions for both temperature and velocity. Therefore these analytical solutions are valid only for relatively high aspect ratio cavities at this Ra for both Pr.

The numerical results and analytical solutions are also compared in Figure 3 for $A=1$ and Rayleigh numbers from $10^{3}$ to $10^{8}$, for $\operatorname{Pr}$ of 0.7 and 7.5. The agreement is poor for the low Rayleigh number cases, particularly for the near wall temperature. Good agreement for both temperature and velocity is obtained for $\mathrm{Ra}=10^{7}$. Additional results have been obtained for 


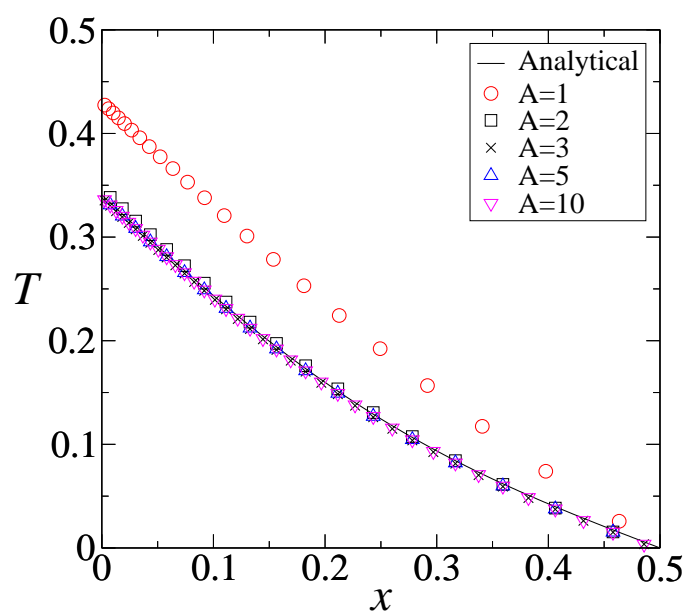

(a)

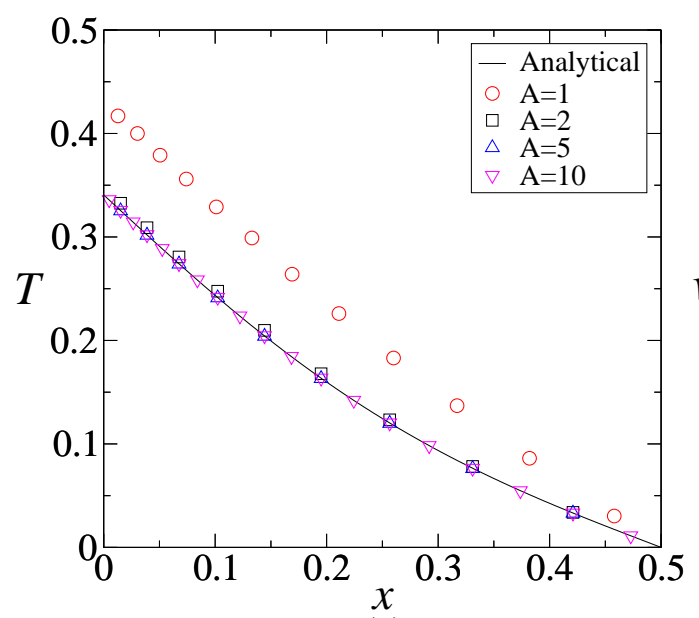

(c)

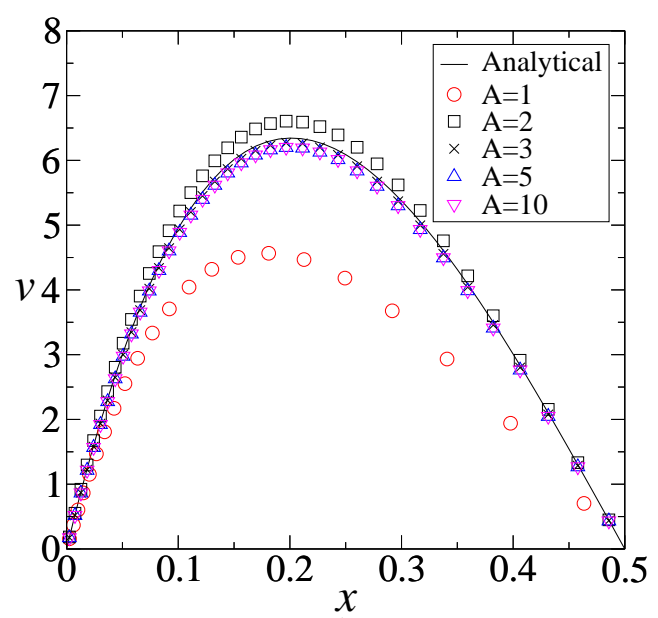

(b)

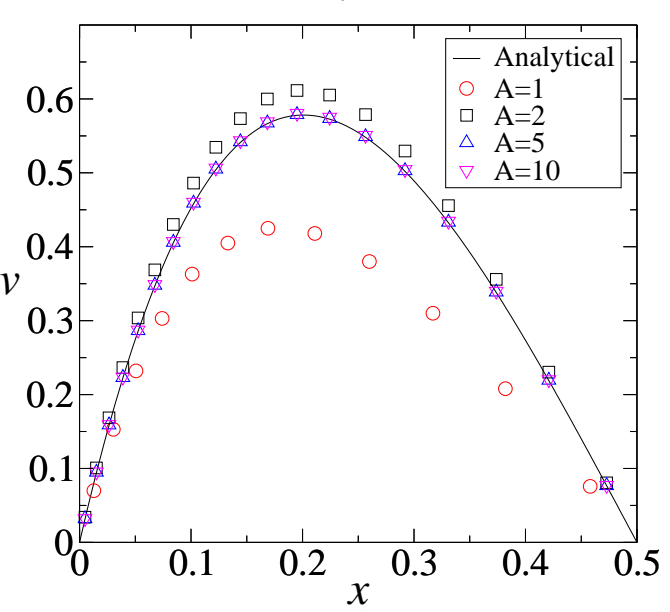

(d)

FiguRE 2: Comparison of analytical and numerical temperature and velocity profiles from the heated wall at $y=A / 2$ for $\mathrm{Ra}=10^{3}$ with various $A$ for $\mathrm{Pr}$ of $0.7(\mathrm{a}, \mathrm{b})$ and $7.5(\mathrm{c}, \mathrm{d})$. 


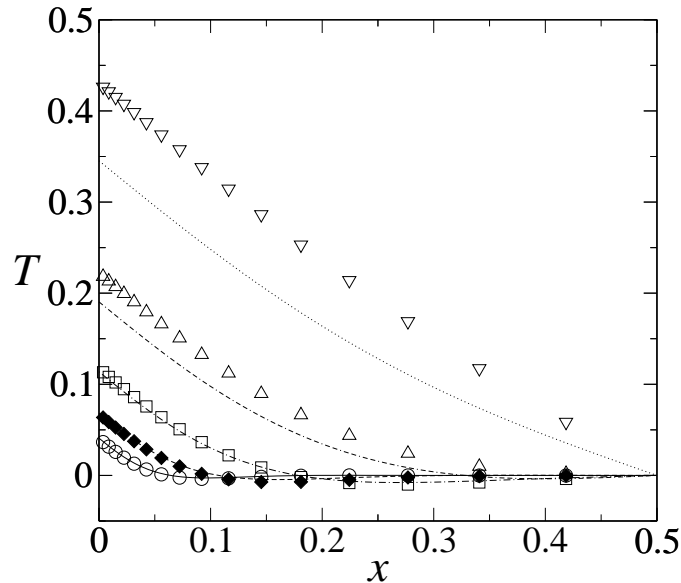

(a)

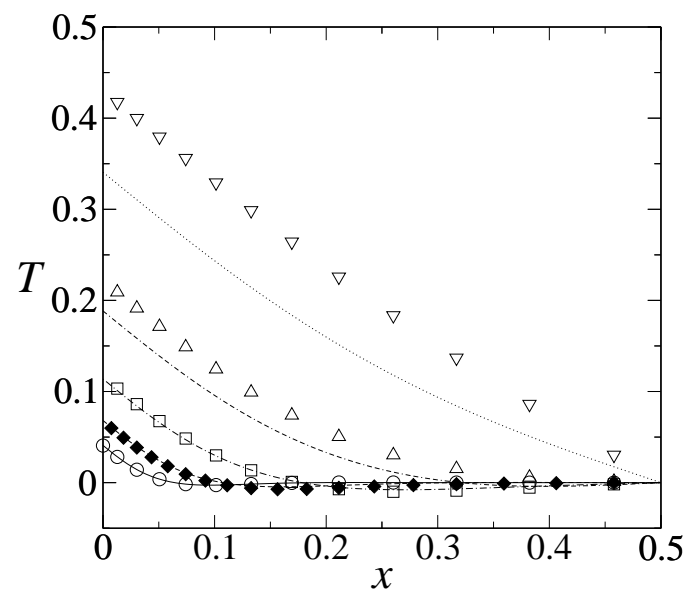

(c)

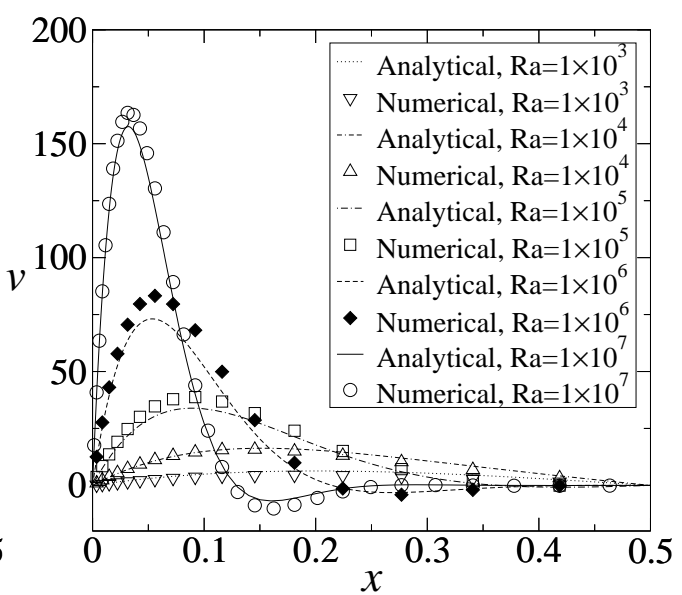

(b)

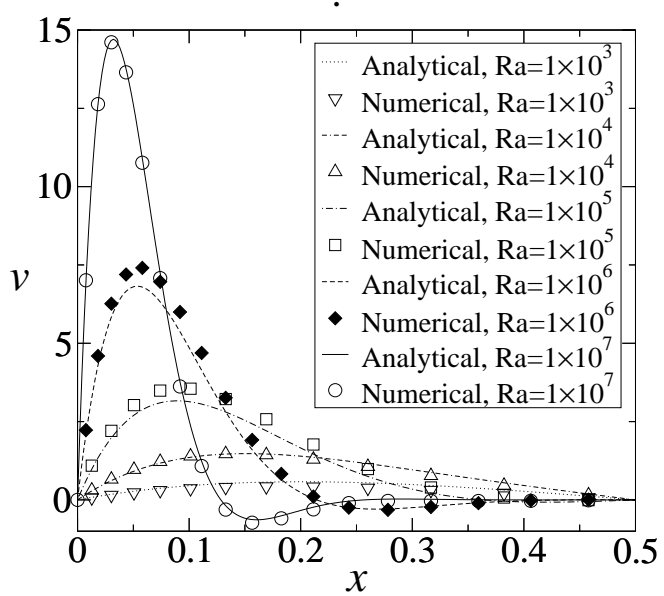

(d)

FiguRE 3: Temperature and velocity profiles near the heated wall in a square cavity at $y=A / 2$ for $\operatorname{Pr}$ of 0.7 (a, b) and 7.5 (c, d) with various Ra, as marked. 


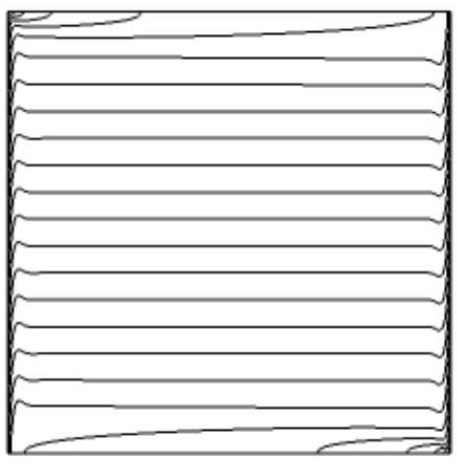

(a)

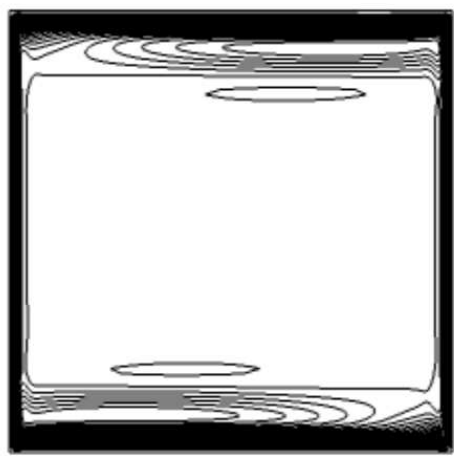

(b)

Figure 4: Isotherms (a) and streamlines (b) of fully developed flow in a square cavity with $\mathrm{Ra}=5.8 \times 10^{9}$ and $\operatorname{Pr}=7.5$.

higher Rayleigh numbers, not shown here, demonstrating very good agreement for temperature and velocity. Figure 4 contains the temperature and stream function contours of the fully developed flow in a square cavity with $\mathrm{Ra}=5.8 \times 10^{9}$ and $\operatorname{Pr}$ of 7.5 . The temperature contours demonstrate that, at full development, the cavity is fully stratified and the stratification contours show that flow occurs only in the vicinity of the boundaries, with a quiescent core [4].

An important parameter for the fully developed flow in a cavity is the stratification. We obtain a prediction for the stratification analytically, for a given Ra, by combining equations (9) and (10). The analytic prediction is compared to the numerical results, where the numerical results is obtained as $\partial T / \partial y$ at the centre of the cavity. Results have been obtained for a range of $\mathrm{Ra}=10^{2}$ to $10^{6}$ with $A=1,2,5$ and 10 for both Pr of 0.7 and 7.5, compared to the analytical solution, shown in Figure 5. Again, the analytical solution does not apply to the low aspect ratio cavities with low Rayleigh number. However, the analytical solution for the square cavity is valid when the Rayleigh number is greater than $10^{6}$, and for $\mathrm{Ra}=10^{3}$ when $A \geq 5$, corresponding to previous results for both Pr. 


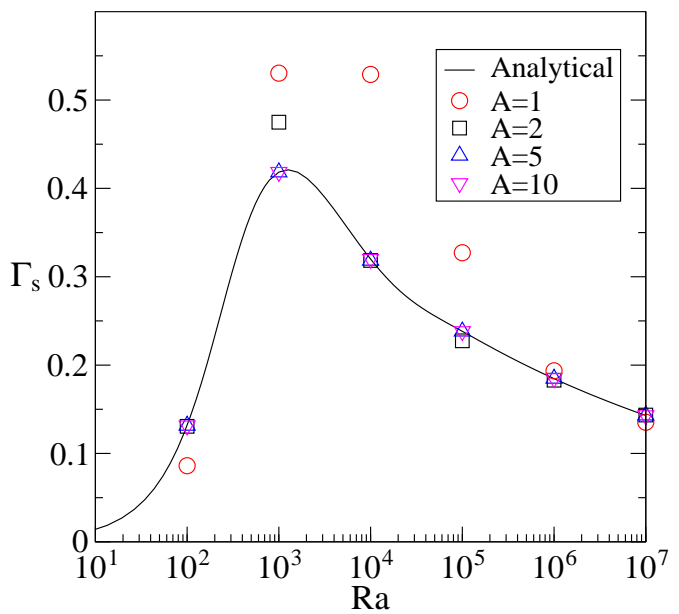

(a)

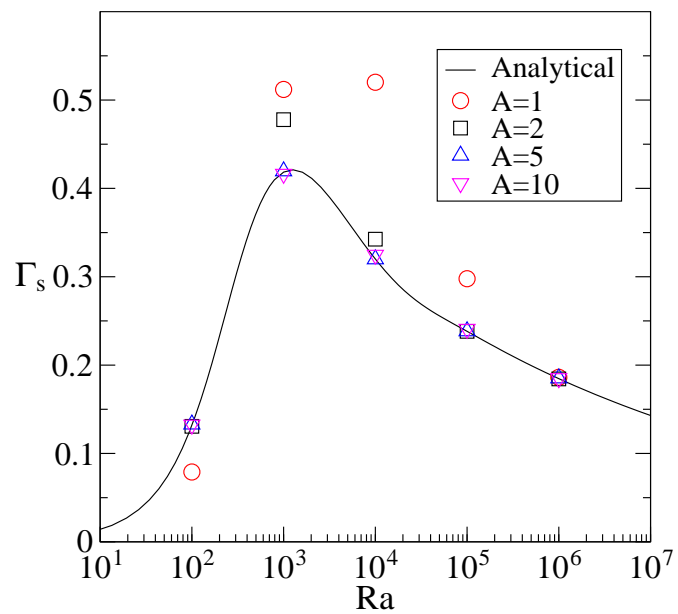

(b)

Figure 5: Background stratification in cavities with various Ra and $A$, for Pr of 0.7 (a) and 7.5 (b).

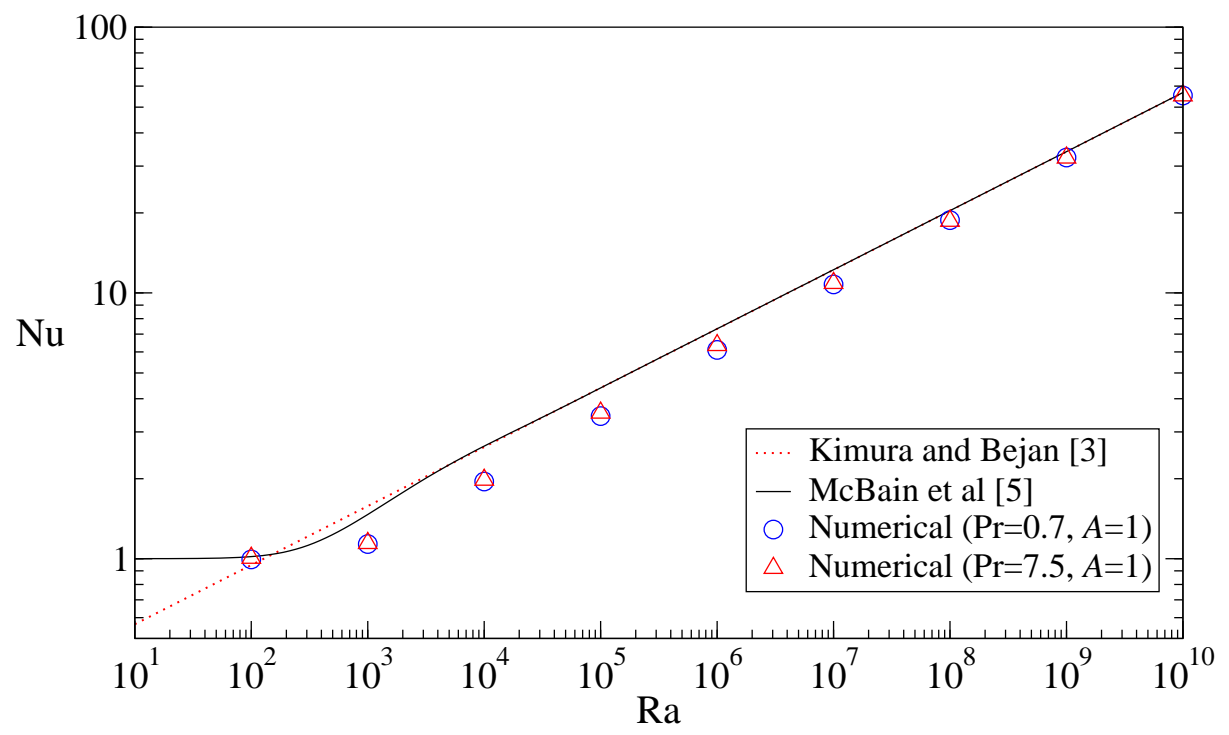

FiguRE 6: Heat transfer relation for the isoflux cavity. 
The Nusselt number $\mathrm{Nu}=\frac{1}{A} \int_{0}^{A} \frac{1}{T(0, y)} d y$ for the square cavity with both $\mathrm{Pr}$ has been investigated and compared to that obtained from the analytical solution in Figure 6. The results confirm that the analytical solution for the evenly heated slot can be applied to the isoflux cavity for high Rayleigh numbers. The numerical results show that Nusselt number is independent of Pr.

\section{Conclusions}

The analytical solution for the evenly heated and cooled slot derived by Lietzke [7], combined with the relation between Ra and $\gamma$ in a cavity $[3,8]$ are compared to numerical solutions with a range of aspect ratios and various Rayleigh numbers. The results show that the analytical solutions are valid for tall cavities (present study, $A \geq 5$ ) over the entire range of Rayleigh numbers considered. However, these solutions also apply to the small aspect ratio cavities with a high enough Rayleigh number, for example, a square cavity with $\mathrm{Ra}=10^{7}$. These results agree well for $\mathrm{Pr}$ of 0.7 and 7.5. The fully developed high Ra square cavity has a constant boundary layer thickness and one dimensional flow, parallel to the side walls away from the floor and ceiling, as seen in Figure 4. When the Rayleigh number is high enough, the floor and ceiling effects are confined to small regions allowing one dimensional flow on the side-walls, even for low aspect ratio cavities. The side wall flow is then represented by the analytical solution, as for high aspect ratio cavities at lower Rayleigh numbers.

\section{References}

[1] Armfeld, S. W., and Patterson, J. C., Direct simulation of wave interactions in unsteady natural convection in a cavity. Int. J. Heat 
Mass Transfer 34 (1991) 929-940. doi:10.1016/0017-9310(91)90004-X C978, C981

[2] Armfeld, S. W., and Patterson, J. C., Wave properties of natural convection boundary layers. J. Fluid Mech. 239 (1992) 195-211. C978, C981

[3] Desrayaud, G., and Nguyen, T. H., Instabilities themoconvectives dans une cavite a flux imposes, in Douzième Congrès Canadien de Mécanique Appliquée (1989) 716-717. C978, C979, C982, C987

[4] Jiracheewanun, S., Armfield, S. W., Behnia, M., and McBain, G. D., The transient behaviour of a differentially heated cavity with isoflux boundaries, Proc. 15th A'asian Fluid Mechanics Conf. (2004) Paper AFMC00246. C978, C985

[5] Kimura, S. and Bejan, A., The boundary layer natural convection regime in a rectangular cavity with uniform heat flux from the side, J. Heat Transfer 106 (No. 1) (1984) 98-103. C978

[6] Leonard, B. P., A stable and accurate convective modeling procedure based on quadratic upstream interpolation, Comp. methods appl. mech. engr. 19 (1979) 59-98. doi:10.1016/0045-7825(79)90034-3 C981

[7] Lietzke, A. F., Theoretical and experimental investigation of heat transfer by laminar natural convection between parallel plates, (NACA, 1955) Report 1223. C979, C981, C987

[8] McBain, G. D., Armfield, S. W. and Jiracheewanun, S., The conduction and convection regimes in a cavity with evenly heated and cooled vertical walls, 8th Australasian Heat and Mass Transfer Conf. (2005). (in press) C982, C987

[9] Norris, S. E. and Armfield, S. W., Solving the Navier-Stokes equations on a workstation cluster, ANZIAM J. 42(E) (2000) C1058-C1075. http://anziamj.austms.org.au/V42/CTAC99/Norr C981 
[10] Patterson, J. C. and Armfield, S. W., Transient features of natural convection in a cavity, J. Fluid Mech. 219 (1990) 469-497. C978, C981

[11] Patterson, J. and Imberger, J., Unsteady natural convection in a rectangular cavity, J. Fluid Mech. 100 (1980) 65-86. C978 


\section{Author addresses}

1. S. Jiracheewanun, School of Aerospace, Mechanical and Mechatronic Engineering, University of Sydney, New South Wales, Australia.

mailto:sujin@aeromech.usyd.edu.au

2. G. D. McBain, School of Aerospace, Mechanical and Mechatronic Engineering, University of Sydney, New South Wales, Australia.

3. S. W. Armfield, School of Aerospace, Mechanical and Mechatronic Engineering, University of Sydney, New South Wales, Australia.

4. M. Behnia, Dean of Graduate Studies, University of Sydney, New South Wales, Australia. 